\title{
DETC2000/DAC-14229
}

\section{METHOD AND CODE FOR THE VISUALIZATION OF MULTIVARIATE SOLIDS}

\author{
Karim Abdel-Malek \\ Department of Mechanical Engineering and \\ Center for Computer Aided Design \\ The University of lowa \\ lowa City, IA 52246 \\ Tel. (319) 335-5676 \\ amalek@engineering.uiowa.edu
}

\author{
Jingzhou Yang \\ Department of Mechanical Engineering and \\ Center for Computer Aided Design \\ The University of lowa \\ lowa City, IA 52246 \\ jyang@engineering.uiowa.edu
}

\begin{abstract}
This paper is devoted to a method and computer code for the automatic visualization of multivariate solids. Example of a multivariate solids arise in computer aided geometric design when a geometric entity is swept in space, where the totality of points touched by the entity is called the swept volume and is characterized by an equation of many parameters. The method and code are presented in an integrated manner and are aimed at providing the reader with a replicable computer algorithm. The formulation for is based on the implicit function theorem; is applicable to the visualization of solids of any number of parameters; and produces the exact boundary representation. Considering the solid as a manifold (possibly with boundaries), it is shown that further stratification of the various submanifolds yields varieties that can be depicted in $\mathbf{R}^{3}$. A measure of the computational complexity is presented to give the reader a sense of robustness of the method. The code is developed using a symbolic manipulator and is presented with a number of examples.
\end{abstract}

\section{INTRODUCTION}

A broadly applicable analytic formulation for the visualization of multivariate solids is presented that is based on a rigorous mathematical approach. The basic problem which this work addresses is the following: It is required to visualize a solid that is characterized by a single equation of three or more parameters parameters (e.g., a surface has two parameters, the sweep of a surface along a curve yields a solid of three parameters, the extrusion of the solid along an axis yield an equation of a solid parameterized in four parameters, etc.). Sweeping in this paper is defined as the motion of a geometric entity along a given path while specifying its orientation. The objective is to find the new solid volume which represents all of the points in space which the object has occupied at some time during the motion. In this paper, we present a method that is comprehensive and that yields a formulation for visualizing the exact solid. The main challenge for this formulation has been the lack (and proclaimed difficulty) of computer implementation. Therefore, the motivation for this work is to demonstrate a simple stepby-step computer program that implements our proposed formulation. While the code represents thousands of lines of computer programs, it is presented in concise and replicable form using a symbolic manipulator.

The topic of multivariate solid visualization has not received considerable attention in the past because of the delicate mathematics associated with its underlying formulation. The need for implementing swept volumes in many disciplines such as manufacturing automation, is what has recently enticed various researchers to address this issue (see Kieffer and Litvin 1991, Abdel-Malek and Yeh 199b, Blackmore, et al. 1997).

This paper presents a mathematical method that employs symbolic manipulation to implement methods adapted from differential geometry and differential topology towards the visualization of multivariate solids. The underlying problem for all application areas addressed herein is characterized by the definition of a configuration space (a manifold, possibly with singularities) for a point in $\mathbf{R}^{n}$, the movement of which is constrained by a series of parameters. The term "manifold" will be used in a non-rigorous fashion for brevity of exposition (although it may have boundaries and singularities).

Simple sweep technology in commercial systems also exists but is limited by the type of swept entity and the number 
of consecutive sweeps. Moreover, approximations are used to represent the resulting swept volume. Indeed, when consecutive sweeps are implemented, the error significantly increases because the result of each individual sweep operation is approximated. The approximation is necessary because of the limitation imposed by current formulations that allow only three parameters (two for the swept entity and one for the sweep path). The main goal of this research is to investigate means and methods for implementing this technology into commercial code in a fast and efficient manner.

We will first review prior work in the field. The background formulation will then be presented in Section 3 is an integrated and systematic manner taking into account both implicitly and parametrically defined geometric entities. The determination of all parts comprising the manifold (manifold stratification) will be presented in the context of determining all varieties of the submanifold (Sections 3-5). Computer implementation and computational complexity of the method will be presented in terms of the processing time required. Illustrative examples will be shown and future directions of this research will be discussed. Future directions of this research will be addressed in Section 12 .

\section{PRIOR RESEARCH}

Trivariate B-Spline and Bezier solids have been treated by a number of researchers. Stanton, et al. (1977), Casale and Stanton (1985), and Farouki and Hinds (1985) all discuss the trivariate form, but avoid the equations of construction of the general boundary surfaces. Lasser (1985) discusses the general trivariate B-spline form, the generation of points in the volume and the generation of derivatives of these solids. Sederberg and Parry (1986) utilized the freeform trivariate B-spline solid for deformations. They embed an object in a deformable region of space defined by the trivariate solid such that each point of the object has a unique parameterization that defines its position in the region. The trivariate region is then altered by moving its control points. Using the trivariate form gives great flexibility to the definition of the deformable regions, and gives few parameters, which can be used to control the deformation. Rappaport, et al. (1996) have incorporated physical properties into freeform trivariate solids. Reus, et al. (1992) treated the trivariate tensor-product solid in a physical manner.

The most rigorous work pertaining to the determination of the boundary of swept volumes is the use of sweep differential techniques (Blackmore and Leu 1992, Blackmore, et al. 1992, Weld and Leu 1991, and Leu, et al. 1986). In this work, the sweep differential equation (called SDE) and the boundary flow methods are shown to be related to the Lie group theory structure of Euclidean motions. The extension of the sweep differential equation method is also used to determine deformed sweeps (Blackmore, et al. 1994). This work presents an elegant method for determining the swept volume with applications to robotics and to $\mathrm{NC}$ verification
(Blackmore, et al. 1997a and Leu, et al. 1997). A summary of this work presented by the authors can be found in Blackmore, et. al (1997b).

Other formulations for addressing the sweep generated by deformable objects was reported by Boussac and Crosnier (1996). Because of the importance of the NC verification problem, there have been many works addressing simulation technologies such as those reported by Menon and Voelker (1992), Liang, et al. (1997), Liu, et al. (1996), Jerard and Drysdale (1988), Voelker and Hunt (1985). Envelope theory has met with limited success at evaluating the swept volume as first presented by Wang and Wang (1988) and Sambandan and Wang (1989), and followed by many reports extending the theory. For example, Martin and Stephenson (1990) evaluated the swept volume of a three-dimensional object moving along an arbitrary path and have highlighted the implementation of the swept volume technique as an additional tool in Constructive Solid Geomtery (CSG). To date, the envelope theory has not been shown to solve consecutive sweeping with more than three parameters, nor has it been extended to determine voids in the volume. Other reports that have addressed the sweeping of objects in space include Klok (1986), Ahn, et al. (1993), Bronsvoort, et al. (1989), and Coquillart (1987), Ilies and Shapiro (1999), Juttler and Wagner (1996 and 1999).

Expanding the "domain of solid modeling using swept volumes" has also been asserted by researchers to be of great importance (Hu and Ling 1996 and Ganter et al. 1993). Learning and creating new methods to represent complex solid models is a goal that is of importance to many industries that implement CAD technology.

Kieffer and Litvin (1991) developed an algorithm for the determination of all surfaces and edges that may bound the swept volume. Computations of volumes swept by polyhedral objects in order to compute viewpoints for monitoring objects and features in an active robot work-cell is reported by Abrams and Allen (1995). Another type of mathematical approach is defined by Schroeder and Lorensen (1994) as the minimization of the sum of distances from the moving object (a polygon). The instantaneous screw theory, adopted from kinematics, is also used to generate a swept volume (Parida and Mudur 1994). More recently, sweep techniques were used to define boundaries of trivariate solids for visualization purposes (Joy and Duchaineau 1999 and Madrigal and Joy 1999).

In prior work, we have demonstrated (Abdel-Malek and Yeh 1997a, Abdel-Malek, et al. 1997) a general formulation for identifying singular surfaces that may exist on the boundary of a swept volume. The method was implemented to robotics analysis (Abdel-Malek and Yeh 1997b, Abdel-Malek, et al. 1999a) and to NC verification (Abdel-Malek, et al. 1999b). Recently, it was shown that a quadratic form on a surface characterizing the acceleration of a point in the volume identifies the boundary (Abdel-Malek, et al. 1998) of the volume in a systematic manner. Furthermore, a methodology 
was developed using a formulation adapted from kinematics (called the Denavit-Hartenberg representation method) to accurately and systematically represent consecutive sweeps (Abdel-Malek and Othman 1999, Blackmore, et al. 1999, Abdel-Malek, et al. 1999c).

\section{FORMULATION FOR IMPLICIT AND PARAMETRIC SOLIDS}

The purpose of this section is to present the underlying formulation in an integrated manner characterizing the sweep of both implicitly and parametrically defined surfaces to generate solids, by means of defining the manifold with singularities, performing a manifold stratification, and determining all varieties and visualizing manifold strata. We present the complete formulation in a referenced manner pertinent to definitions and theorems adopted from Differential Geometry and Differential Topology (Spivak 1976, Guillemin and Pollack 1974, Lu 1976).

Consider a geometric entity parametrized in terms of one or more variables as a $(3 \times 1)$ vector given by $\boldsymbol{\Gamma}(\mathbf{u})$, where $\mathbf{u}=\left[u_{1}, \ldots, u_{n}\right]^{T}$ in $n$-dimensional space $\mathbf{R}^{n}$. This could be a curve, a surface or volume such as the sphere $\left.\Gamma(\mathbf{u})=\left[\begin{array}{lll}u_{1} \cos u_{2} \cos u_{3} & u_{1} \cos u_{2} \sin u_{3} & u_{1} \sin u_{2}\end{array}\right]^{T}\right)$.

In order to generalize the formulation, we consider boundaries imposed on $\boldsymbol{\Gamma}(\mathbf{u})$ in the form of constraints on the parameters $u_{i}$ characterized by inequalities in the form of $u_{i}^{L} \leq u_{i} \leq u_{i}^{U}$.

We will also consider surfaces implicitly defined on $K$ where $\Gamma(\mathbf{u})=\left[\begin{array}{lll}u_{1} & u_{2} & u_{3}\end{array}\right]^{T}$ and where this surface will be represented by a number of inequalities, with no restriction on the number of inequalities nor on the type of variables. Let $K$ be represented by

$$
\begin{aligned}
& K:=\left\{\mathbf{u}: \ell_{1}^{L} \leq f_{1}(\mathbf{u}) \leq \ell_{1}^{U}, \ldots, \ell_{N}^{L} \leq f_{N}(\mathbf{u}) \leq \ell_{N}^{U},\right. \\
& \left.u_{1}^{L} \leq u_{1} \leq u_{1}^{U}, \ldots, u_{M}^{L} \leq u_{M} \leq u_{M}^{U}\right\}
\end{aligned}
$$

where $\mathbf{f}=\left[f_{1}, \ldots, f_{N}\right]^{T}$ and $f_{i}(\mathbf{u})$ denotes an expression representing the surface; $\ell_{i}$ is a limit; the upper subscript $\mathrm{L}$ indicates the lower limit; $\mathrm{U}$ denotes the upper limit; $N$ is the number of expressions; and $M$ is the number of variables.

The sweep of a geometric entity is governed by an orientation matrix $\mathbf{R}$ and a path. Each path will be considered as a second geometric entity parametrized in terms of one or more variables as a $(3 \times 1)$ vector $\Psi_{1}\left(v_{1}\right)$. This entity also has a boundary defined by $v_{1}^{L} \leq v_{1} \leq v_{1}^{U}$. The manifold (possibly with corners, generated by the sweep of $\Gamma\left(u_{1}, \ldots, u_{n}\right)$ on $\Psi_{1}\left(v_{1}\right)$ is defined by the vector

$$
\mathbf{N}_{1}(\mathbf{q})=\mathbf{R}_{1}\left(v_{1}\right) \Gamma(\mathbf{u})+\Psi_{1}\left(v_{1}\right)
$$

where $\mathbf{q}$ is a subset of the Euclidean defined by $\mathbf{q}=\left[\begin{array}{lll}q_{1} & \ldots & q_{n}\end{array}\right]^{T}=\left[\begin{array}{llll}u_{1} & \ldots & u_{n} & v_{1}\end{array}\right]^{T}$ and $\mathbf{R}_{1}\left(v_{1}\right)$ is the $(3 \times 3)$ rotation matrix defining the orientation of the swept entity. In fact, $\mathbf{N}_{1}(\mathbf{q})$ characterizes the set of all points that belong to the manifold. Another sweep motion yields an expanded swept volume in the form of $\mathbf{N}_{2}(\mathbf{q})=\mathbf{R}_{2}\left(v_{2}\right) \mathbf{N}_{1}(\mathbf{q})+\Psi_{2}\left(v_{2}\right)=\mathbf{R}_{2} \mathbf{R}_{1} \Gamma+\mathbf{R}_{2} \Psi_{1}+\Psi_{2}$ where now $\mathbf{q}=\left[\begin{array}{lllll}u_{1} & \ldots & u_{n} & v_{1} & v_{2}\end{array}\right]^{T}$. The generalized case yields a space characterized by the vector function $\xi$, such that

$\boldsymbol{\xi}(\mathbf{q})=\prod_{i=1}^{n+m} \mathbf{R}_{i} \Gamma+\sum_{j=1}^{n+m}\left(\prod_{i=j+1}^{n+m-1}\left[\mathbf{R}_{i}\right] \boldsymbol{\Psi}_{j}\right)+\boldsymbol{\Psi}_{n+m}$

where $\quad \mathbf{q}=\left[\begin{array}{ll}\mathbf{u}^{T} & \mathbf{v}^{T}\end{array}\right]^{T}, \quad \mathbf{u}=\left[\begin{array}{lll}u_{1} & \ldots & u_{n}\end{array}\right]^{T}, \quad$ and $\mathbf{v}=\left[\begin{array}{lll}v_{1} & \ldots & v_{m}\end{array}\right]^{T}$. Note that $\boldsymbol{\xi}(\mathbf{q})$ is defined on the Euclidean space $\mathbf{R}^{n+m}$ where $\boldsymbol{\xi}: \mathbf{R}^{3} \rightarrow \mathbf{R}^{n+m}$, which is a Manifold possibly with corners. To impose inequality constraints, it was shown (Abdel-Malek and Yeh 1997a) that a constraint of the form $q_{i}^{\min } \leq q_{i} \leq q_{i}^{\max }$ can be transformed into an equation by introducing a new set of generalized coordinates $\lambda_{i}$ such that

$$
q_{i}=a_{i}+b_{i} \sin \lambda_{i} ; \quad i=1, \ldots n+m
$$

where $a_{i}=\left(q_{i}^{\max }+q_{i}^{\min }\right) / 2$ and $b_{i}=\left(q_{i}^{\max }-q_{i}^{\min }\right) / 2$ are the mid-point and half-range, respectively. At a point in the manifold, a vector constraint function $\boldsymbol{\Phi}\left(\mathbf{q}^{*}\right)$ with parametrized inequality constraints is defined as

$$
\boldsymbol{\Phi}\left(\mathbf{q}^{*}\right)=\left[\begin{array}{c}
\boldsymbol{\xi}(\mathbf{q}) \\
u_{i}-0.5\left(u_{i}^{U}+u_{i}^{L}\right)-0.5\left(u_{i}^{U}-u_{i}^{L}\right) \sin \lambda_{i} \\
v_{j}-0.5\left(v_{j}^{U}+v_{j}^{L}\right)-0.5\left(v_{j}^{U}-v_{j}^{L}\right) \sin \lambda_{n+j}
\end{array}\right]
$$

where $i=1, \ldots, n$ and $j=1, \ldots, m$ and where $\mathbf{q}^{*}=\left[\begin{array}{lll}\mathbf{u}^{T} & \mathbf{v}^{T} & \boldsymbol{\lambda}^{T}\end{array}\right]^{T}=\left[\begin{array}{lll}q_{1} & \ldots & q_{2 n+2 m}\end{array}\right]^{T}$ is the vector of all generalized coordinates and $\lambda=\left[\lambda_{1}, \ldots, \lambda_{n+m}\right]^{T}$ is the vector of new generalized coordinates. The vector function $\boldsymbol{\Phi}\left(\mathbf{q}^{*}\right)$ represents every point in the swept volume.

For the implicit formulation, the constraint is defined by

$$
\boldsymbol{\Phi}\left(\mathbf{q}^{*}\right):=\left[\begin{array}{c}
\boldsymbol{\xi}(\mathbf{q}) \\
f_{i}(\mathbf{u})-0.5\left(\ell_{i}^{U}+\ell_{i}^{L}\right)-0.5\left(\ell_{i}^{U}-\ell_{i}^{L}\right) \sin \lambda_{i} \\
u_{j}-0.5\left(u_{j}^{U}+u_{j}^{L}\right)-0.5\left(u_{j}^{U}-u_{j}^{L}\right) \sin \lambda_{N+j} \\
v_{k}-0.5\left(v_{k}^{U}+v_{k}^{L}\right)-0.5\left(v_{k}^{U}-v_{k}^{L}\right) \sin \lambda_{N+n+k}
\end{array}\right]
$$

where $i=1, \ldots, N ; j=1, \ldots, n$; and $k=1, \ldots, m$ where the vector of generalized coordinates has been augmented to 


$$
\begin{aligned}
\mathbf{q}^{*} & =\left[\begin{array}{lll}
x_{1}, \ldots, x_{N}, v_{1}, \ldots, v_{m}, \lambda_{1}, \ldots, \lambda_{N+n+m}
\end{array}\right]^{T} \\
\mathbf{q}^{*} & =\left[\begin{array}{llll}
\mathbf{u}^{T} & \mathbf{v}^{T} & \lambda_{1}^{T} & \boldsymbol{\lambda}_{2}^{T}
\end{array}\right]^{T}, \text { where } \\
\boldsymbol{\lambda}_{1} & =\left[\begin{array}{lll}
\lambda_{1} & \ldots & \lambda_{N}
\end{array}\right]^{T}, \text { and } \boldsymbol{\lambda}_{2}=\left[\begin{array}{llll}
\lambda_{N+1} & \ldots & \lambda_{N+n+m}
\end{array}\right]^{T} .
\end{aligned}
$$

\section{IMPLICIT FUNCTION THEOREM}

In order to have a well-posed formulation, constraints that are used to model the geometry of this problem should be independent, except at certain critical surfaces in the manifold where the Jacobian becomes singular. However, the sweep Jacobian is not square. It is important, therefore, that there not be open sets in the space of the generalized parameters in which the constraints are redundant. At any interior point of the constraint space $\mathbf{q}^{*}$, the sweep Jacobian for parametric sweeps is defined as

$\boldsymbol{\Phi}_{\mathbf{q}^{*}}=\left[\begin{array}{c:c}\boldsymbol{\xi}_{\mathbf{q}} & \mathbf{0} \\ \hdashline \mathbf{I} & \mathbf{q}_{\lambda}\end{array}\right]$

where $\mathbf{q}_{\boldsymbol{\lambda}}=\partial \mathbf{q} / \partial \boldsymbol{\lambda}$ and $\boldsymbol{\xi}_{\mathbf{q}}=\partial \boldsymbol{\xi} / \partial \mathbf{q}$. For implicit sweeps, the Jacobian is derived as

$\boldsymbol{\Phi}_{\mathbf{q}^{*}}=\left[\begin{array}{ccc}\xi_{\mathbf{q}} & \mathbf{0} & \mathbf{0} \\ \mathbf{f}_{\mathbf{q}} & \mathbf{f}_{\lambda_{1}} & \mathbf{0} \\ \mathbf{I} & \mathbf{0} & \mathbf{q}_{\lambda_{2}}\end{array}\right]$

where $\mathbf{f}_{\mathbf{q}}=\partial \mathbf{f} / \partial \mathbf{q} ; \mathbf{f}_{\boldsymbol{\lambda}_{1}}=\partial \mathbf{f} / \partial \boldsymbol{\lambda}_{1} ;$ and $\mathbf{q}_{\boldsymbol{\lambda}_{2}}=\partial \mathbf{q} / \partial \boldsymbol{\lambda}_{2}$. Since the swept entities are smooth and continuously differentiable, then the sweep Jacobian exists for both parametrically and implicitly defined surfaces. In both cases, if the rank of the mapping $\boldsymbol{\xi}_{\mathbf{q}}$ is maximal, i.e. 3, the image under $\boldsymbol{\Phi}$ of a small neighborhood of $\mathbf{q}^{*}$ will be a threemanifold in three-space. At a point $\mathbf{q}^{*}$ where rank of $\boldsymbol{\Phi}_{\mathbf{q}^{*}}$ is less than 3, the sweep Jacobian becomes singular, or rankdeficient. One might describe this deficiency as "redundancy" in that some number of parameters in the constraint space become dependent, possibly lowering the dimension of the image set. Redundancy occurs when the Jacobian $\boldsymbol{\Phi}_{\mathbf{q}}$ is rankdeficient. Candidate varieties that are boundary to the manifold are identified by computing values that create a locus of points where the Jacobian has lower rank than maximal. Varieties are computed when the locus of points has a Jacobian with a lower rank. Let $\partial W$ characterize a manifold with corners as

$$
\partial W \subset\left\{\operatorname{Rank} \boldsymbol{\Phi}_{\mathbf{q}^{*}}\left(\mathbf{q}^{*}\right) \leq \alpha, \text { for some } \mathbf{q}^{*}\right\}
$$

where the rank is at most $(n+m+3)$ for parametric and $(n+m+N+3)$ for implicit sweeps.

\section{VARIETIES}

In order to enforce the implicit function theorem, square subjacobians are determined. For example, a $3 \times 4$ Jacobian and a rank deficiency of one, there are four $3 \times 3$ subjacobians. Equating the determinants of the subjacobians to zero forms analytic functions. The set of common zeros defined by this finite number of analytic functions are called varieties. In the case of implicit surfaces, these varieties are readily computed and visualized. In the case of parametric entities, the rank deficiency conditions yield analytic functions equated to zero that must be simultaneously solved. The solutions to the set of functions are singular sets defined by the vector $\mathbf{p}_{i}$, where $\mathbf{q}^{T}=\left[\begin{array}{ll}\mathbf{p}_{i}^{T} & \mathbf{d}^{T}\end{array}\right]$, i.e., $\mathbf{d}$ contains the remaining non-constant parameters.

Substituting $\mathbf{p}_{i}^{T}$ into $\boldsymbol{\xi}(\mathbf{q})$ yields strata denoted by $V_{i}$ as

$$
V_{i}: \xi(\mathbf{d})=\boldsymbol{\xi}\left(\mathbf{q}, \mathbf{p}_{i}\right) \quad i=1, \ldots, \beta
$$

Where $\beta$ is the number of varieties. In the case of implicitly defined entities, the vector $\boldsymbol{\xi}(\mathbf{d})$ is accompanied by the associated set of functions $\mathbf{f}=\left[f_{1}, \ldots, f_{N}\right]^{T}$. Note that the stratification process is to decompose a manifold (Eq. 10) into a finite disjoint (or union) of closed submanifolds such that $\partial W=V_{1} \cap V_{2} \cap \ldots \cap V_{\beta}$

While some strata can be further decomposed, it is only necessary to stratify those that cannot readily be visualized.

Some of these varieties are $\in \mathbf{R}^{3}$ and are rank deficient. This indicates that the resulting submanifold can be further stratified and is composed of many parts (called strata). The stratification process is to decompose a variety $V$ into a finite, disjoint union or closed manifolds.

\section{MANIFOLD STRATIFICATION}

For the case when the stratum $\boldsymbol{\xi}(\mathbf{d})$ cannot be visualized, where $\boldsymbol{\xi}_{i}\left(\mathbf{d}_{i}\right): \mathbf{d}_{i}(\boldsymbol{\lambda}) \in \mathbf{R}^{3} \rightarrow \mathbf{R}^{3}$, and where $\boldsymbol{\lambda}$ is the corresponding vector of slack variables $\lambda=\left[\begin{array}{lll}\lambda_{k} & \lambda_{\ell} & \lambda_{m}\end{array}\right]^{T}$, the Jacobian of the variety is $\xi_{\lambda}=\xi_{\mathrm{d}} \mathbf{d}_{\lambda}$ where $\xi_{\mathbf{d}}=\partial \xi / \partial \mathbf{d}$ and $\mathbf{d}_{\lambda}=\partial \mathbf{d} / \partial \boldsymbol{\lambda}$. For a parameter at its limit $\left(q_{i}^{\text {limit }}\right)$, the second block matrix of Eq. (13) is rank deficient. An elementary matrix of row operations $\mathbf{E}_{i}$ applied to $\mathbf{d}_{\lambda}$ yields a row echelon form such that

$\mathbf{E}_{i}\left[\mathbf{d}_{\lambda}\right]=\mathbf{E}_{R E}$

where $\quad \mathbf{E}_{1}=\left[\begin{array}{ccc}0 & 1 & 0 \\ 0 & 0 & 1 \\ 0 & 0 & 0\end{array}\right], \quad \mathbf{E}_{2}=\left[\begin{array}{ccc}1 & 0 & 0 \\ 0 & 0 & 1 \\ 0 & 0 & 0\end{array}\right], \quad$ and $\quad \mathbf{E}_{3}=\left[\begin{array}{ccc}1 & 0 & 0 \\ 0 & 1 & 0 \\ 0 & 0 & 0\end{array}\right]$; where the subscript denotes the parameter number, and $\mathbf{E}_{R E}$ is a row echelon form. This same matrix applied to $\boldsymbol{\xi}_{\mathbf{d}}{ }^{T}$ yields 
$\mathbf{E}_{i}\left[\boldsymbol{\xi}_{\mathbf{d}}\right]^{T}=\left[\begin{array}{l}\Lambda \\ \mathbf{0}\end{array}\right]$

where $\Lambda=\left[\begin{array}{lll}\Lambda_{1} & \Lambda_{2} & \Lambda_{3}\end{array}\right]$

where $\operatorname{dim}(\Lambda)=(2 \times 3)$ and $\operatorname{dim}\left(\Lambda_{j}\right)=(2 \times 1)$ for $j=1,2,3$.

For $\Lambda$, the application of the implicit function theorem yields three analytic functions that are equated to zero as

$\left|\boldsymbol{\Lambda}_{1} \boldsymbol{\Lambda}_{2}\right|=0 ; \quad\left|\boldsymbol{\Lambda}_{2} \boldsymbol{\Lambda}_{3}\right|=0 ; \quad$ and $\left|\boldsymbol{\Lambda}_{1} \boldsymbol{\Lambda}_{3}\right|=0$

Simultaneous solutions to the three equations are the varieties of this submanifold, i.e., a second stratification process (see Section A.18). This process can be repeated for each resulting stratum that cannot be visualized.

\section{COMPUTER IMPLEMENTATION}

In this section, we illustrate the implementation of the above theoretical framework into a computer program that automatically performs the analysis and visualization of multivariate solids. Mathematica ${ }^{\circledR}$ was used to develop this code and to visualize the volume. For commercial applications, it is recommended that this be converted into a more efficient basic language or embedded in hardware. In order to keep the code listing to a minimum, the presentation will adhere to tasks that are vital to the completion of the algorithm.

\begin{tabular}{|c|}
\hline Mathematica ${ }^{\circledR}$ code \\
\hline $\begin{array}{l}\text { I. Definitions } \\
\text { qL1=0; qU1=5Pi/4; qL2=-Pi/4; qU2=3Pi/4; qL3=0; } \\
\text { qU3=5Pi/4; qL4=0; qU4=2Pi; }\end{array}$ \\
\hline 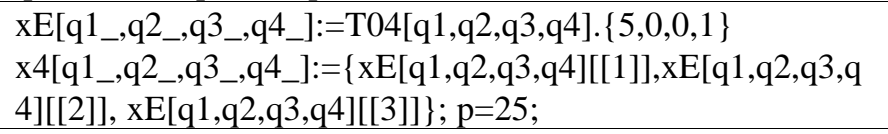 \\
\hline 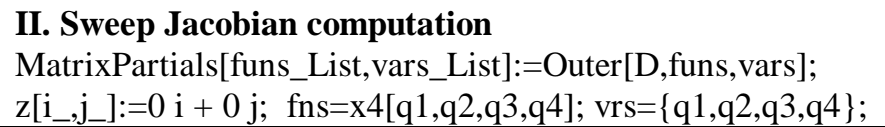 \\
\hline $\begin{array}{l}\text { III. Computing the square sub-jacobians } \\
\text { Mm2=Table[Simplify[Minors[MatrixPartials[fns,vrs],3]]]; }\end{array}$ \\
\hline $\begin{array}{l}\text { IV. Determination of Varieties } \\
\text { a2=Solve[\{Minors[MatrixPartials[fns,vrs],3]== } \\
\quad \text { Array }[z,\{1,4\}]\},\{\mathrm{q} 2\}]\end{array}$ \\
\hline 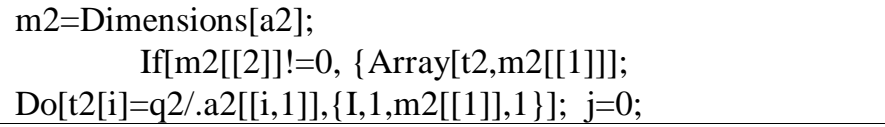 \\
\hline 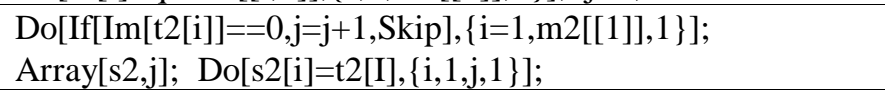 \\
\hline $\operatorname{Do}[\operatorname{If}[\mathrm{s} 2[\mathrm{i}]==0, \mathrm{~s} 2[\mathrm{j}+1]=\mathrm{Pi}, \mathrm{No}],\{\mathrm{i}, 1, \mathrm{j}, 1\}]$ \\
\hline $\begin{array}{l}\mathrm{qs} 2=\operatorname{Cases}\left[\{\mathrm{s} 2[\mathrm{i}],\{\mathrm{i}=1, \mathrm{j}+1,1\}\}, \mathrm{s} 2 \_/ \mathrm{s} 2<=\mathrm{qU} 2 \& \& \mathrm{~s} 2>=\mathrm{qL} 2\right] ; \\
\mathrm{d} 2=\text { Dimension[qs2]; }\end{array}$ \\
\hline $\begin{array}{l}\text { V. Compute the stratum for each variety } \\
\text { If[d2[[1]]>=1, }\{\mathrm{g}[\mathrm{p}]:=\text { ParametricPlot } 3 \mathrm{D}[\mathrm{x} 4[\mathrm{q} 1, \mathrm{qs} 2[[1]], \mathrm{q} 3, \mathrm{q} \\
\mathrm{L} 4],\{\mathrm{q} 1, \mathrm{qL} 1, \mathrm{qU} 1\},\{\mathrm{q} 3, \mathrm{qL} 3, \mathrm{qU} 3\}, \text { Compiled->False], } \\
\quad \mathrm{p}=\mathrm{p}+1\}, \mathrm{No}] \text {; } \\
\text { If }[\mathrm{d} 2[[1]]>=1,\{\mathrm{~g}[\mathrm{p}]:=\text { ParametricPlot3D[x4[q1,qs2[[1]],q3,q }\end{array}$ \\
\hline
\end{tabular}

U4], \{q1,qL1,qU1\},\{q3,qL3,qU3\},Compiled->False], $\mathrm{p}=\mathrm{p}+1\}, \mathrm{No}]$;

If $[\mathrm{d} 2[[1]]>=1,\{\mathrm{~g}[\mathrm{p}]:=$ ParametricPlot3D[x4[q1,qs2[[1]],qL3, q4], \{q1,qL1,qU1\},\{q3,qL3,qU3\},Compiled->False], $\mathrm{p}=\mathrm{p}+1\}, \mathrm{No}]$;

If[d2[[1]] $>=1,\{\mathrm{~g}[\mathrm{p}]:=$ ParametricPlot3D[x4[q1,qs2[[1]],qU3, q4], \{q1,qL1,qU1\},\{q3,qL3,qU3\},Compiled->False], $\mathrm{p}=\mathrm{p}+1\}, \mathrm{No}] ; \ldots$

[Repeat above procedures with respect to other variables and save the graphics of each singular set]

\section{Manifold stratification}

xx1[q2_,q3_,q4_]:=x4[qL1,q2,q3,q4];

Fns $=x x 1[\mathrm{q} 2, \mathrm{q} 3, \mathrm{q} 4]$;

$\mathrm{Vrs}=\{\mathrm{q} 2, \mathrm{q} 3, \mathrm{q} 4\}$;

a11=Solve[ $\{$ Minors[MatrixPartials[fns,vrs],3]==

Array[z, $\{1,1\}]\},\{\mathrm{q} 2\}] ; \mathrm{m} 11=$ Dimensions[a11];

If additional singularities exist, repeat step II to find out the singularities which are in the constraint limits save its resulting graphics in the array $\mathrm{g}[\mathrm{p}]$.

Repeat above procedures to plot the reduced rank deficiency corresponding to qU1, qL2,qU2,.., qL4,qU4

\section{Evaluating the strata of rank deficiency equal to 2}

$\mathrm{g}[1]:=$ ParametricPlot3D[x4[qL1,qL2,q3,q4], $\{\mathrm{q} 3, \mathrm{qL} 3, \mathrm{qU} 3\}$, $\{q 4, q L 4, q U 4\}$, Compiled->False];

$\mathrm{g}[2]:=$ ParametricPlot3D[x4[qL1,qU2,q3,q4], \{q3,qL3,qU3\},

$\{\mathrm{q} 4, \mathrm{qL} 4, \mathrm{qU} 4\}$, Compiled->False $] ; \ldots \ldots .$.

$\mathrm{g}[24]:=$ ParametricPlot3D[x4[q1,q2,qU3,qL4], \{q1,qL1,qU1\}

, \{q2,qL2,qU2\}, Compiled->False];

\section{Visualization of the final swept volume}

Show[Table[g[k],\{k,1,p-1,1\}]]

\section{ILLUSTRATIVE EXAMPLES}

1. Consider a solid defined by

$K:=\left\{\mathbf{u}: \frac{u_{3}^{2}}{16}=\frac{\left(u_{1}-3\right)^{2}}{9}+\frac{u_{2}^{2}}{4}, 0 \leq u_{1} \leq 6,-2 \leq u_{2} \leq 2\right\}$

Note that this is a surface in $\mathbf{R}^{3}$ given by $\frac{z^{2}}{16}=\frac{(x-3)^{2}}{2}+\frac{y^{3}}{4}$. The sweep is defined by the rotation $\mathbf{R}\left(v_{1}\right)=\left[\begin{array}{ccc}\cos \left(\pi v_{1}\right) & -\sin \left(\pi v_{1}\right) & 0 \\ \sin \left(\pi v_{1}\right) & \cos \left(\pi v_{1}\right) & 0 \\ 0 & 0 & 1\end{array}\right] ;$ for $0 \leq v_{1} \leq 2$, where every point in the swept volume is given by $\boldsymbol{\xi}=\boldsymbol{\Psi}\left(v_{1}\right)+\mathbf{R}\left(v_{1}\right)\left[\begin{array}{lll}u_{1} & u_{2} & u_{3}\end{array}\right]^{T}$ and where there will be no translation $\Psi=\left[\begin{array}{lll}0 & 0 & 0\end{array}\right]^{T}$. The parameters are changed to generalized coordinates defined by the vector $\mathbf{q}=\llbracket \begin{array}{llll}q_{1} & q_{2} & q_{3} & q_{4}\end{array} \rrbracket^{T}$ where 
$q_{3}=\operatorname{Sqrt}\left[16\left(\frac{\left(q_{1}-3\right)^{2}}{9}+\frac{q_{2}^{2}}{4}\right)\right]$

$0 \leq q_{1} \leq 6$, and $-2 \leq q_{2} \leq 2$

$\mathbf{R}\left(q_{4}\right)=\left[\begin{array}{ccc}\cos \left(\pi q_{4}\right) & -\sin \left(\pi q_{4}\right) & 0 \\ \sin \left(\pi q_{4}\right) & \cos \left(\pi q_{4}\right) & 0 \\ 0 & 0 & 1\end{array}\right] ; 0 \leq q_{4} \leq 2$

The vector function of Eq. (7) describing the sweep with unilateral constraints after parametrization is written as

$$
\boldsymbol{\Phi}\left(\mathbf{q}^{*}\right)=\left[\begin{array}{c}
q_{1} \cos \left(\pi q_{4}\right)-q_{2} \sin \left(\pi q_{4}\right) \\
q_{2} \cos \left(\pi q_{4}\right)+q_{1} \sin \left(\pi q_{4}\right) \\
q_{3} \\
q_{3}-\operatorname{Sqrt}\left[16 *\left(\frac{\left(q_{1}-3\right)^{2}}{9}+\frac{q_{2}^{2}}{4}\right)\right] \\
q_{1}-3-3 \sin q_{5} \\
q_{2}-2 \sin q_{6} \\
q_{4}-1-\sin q_{7}
\end{array}\right]
$$

where $\mathbf{q}^{*}=\left[\begin{array}{lll}q_{1} & \ldots & q_{7}\end{array}\right]^{T}$. Block matrices of the sweep Jacobian are

$\boldsymbol{\xi}_{\mathbf{q}}=\left[\begin{array}{cccc}\cos \pi q_{4} & -\sin \pi q_{4} & 0 & -\pi q_{2} \cos \pi q_{4}-\pi q_{1} \sin \pi q_{4} \\ \sin \pi q_{4} & \cos \pi q_{4} & 0 & \pi q_{1} \cos \pi q_{4}-\pi q_{2} \sin \pi q_{4} \\ 0 & 0 & 1 & 0\end{array}\right]$

$\left[\begin{array}{ll}\mathbf{f}_{q_{1}} & \mathbf{f}_{\lambda_{1}}\end{array}\right]=\left[\begin{array}{llll}\frac{-4\left(q_{1}-3\right)}{9 \operatorname{Sqrt}\left[\frac{1}{9}\left(q_{1}-3\right)^{2}+\frac{q_{2}^{2}}{4}\right]} & \frac{-q_{2}}{\operatorname{Sqrt}\left[\frac{1}{9}\left(q_{1}-3\right)^{2}+\frac{q_{2}^{2}}{4}\right]} & 1 & 0\end{array}\right]$

Varieties are computed as $\mathbf{p}_{1}, \ldots, \mathbf{p}_{7}$, where $\mathbf{p}_{1}=\left\{q_{5}=\frac{\pi}{2}\right\}$;

$\mathbf{p}_{2}=\left\{q_{5}=-\frac{\pi}{2}\right\} ; \quad \mathbf{p}_{3}=\left\{q_{6}=\frac{\pi}{2}\right\} ; \quad \mathbf{p}_{4}=\left\{q_{6}=-\frac{\pi}{2}\right\} ;$

$\mathbf{p}_{5}=\left\{q_{7}=\frac{\pi}{2}\right\} \quad ; \mathbf{p}_{6}=\left\{q_{7}=-\frac{\pi}{2}\right\} ; \mathbf{p}_{7}=\left\{q_{2}=0\right\}$. In order

to visualize the strata, we substitute each variety into $\boldsymbol{\xi}(\mathbf{q})$ as shown in Fig. 1.

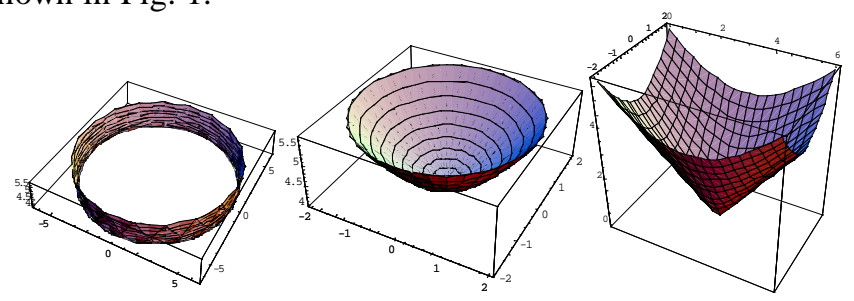

Fig. 1 (a) Stratum due to $\mathbf{p}_{1}$ (b due to $\mathbf{p}_{2}$ (c) due to $\mathbf{p}_{5}$ The solid is shown in Fig. 2.

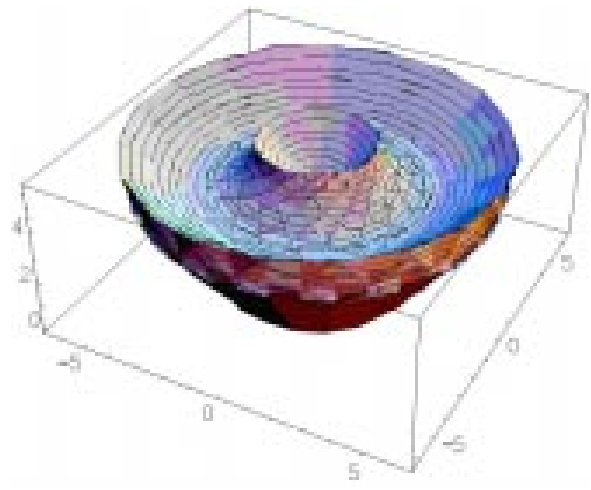

Fig. 2 A 4-parameter solid

\section{COMPUTATIONAL TIME}

The strength of the presented formulation is evidenced in the ability to obtain exact solutions for any number of sweep parameters and for both implicitly and parametrically defined entities. The code written in Mathematica ${ }^{\circledR}$ can be rendered efficient by converting it in a lower level programming language or embedded in hardware. Nevertheless, and to give the reader a sense of computational complexity, we have attempted to measure the computational (CPU) time required for the various examples. Furthermore, a relatively high computational cost is associated with obtaining manifold strata. This is only available when varieties with more than one-to-one mapping occur. The following examples were computed using a Pentium II PC with 64 MB RAM running at $300 \mathrm{MHz}$.

Examples with $n+m=4$

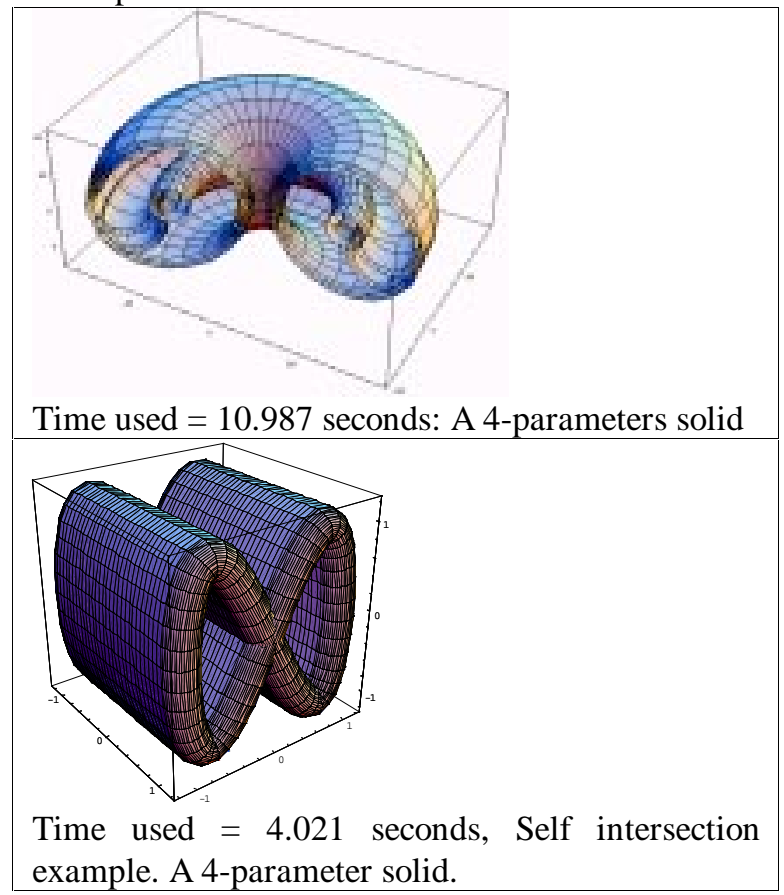


Examples with $n+m=5$

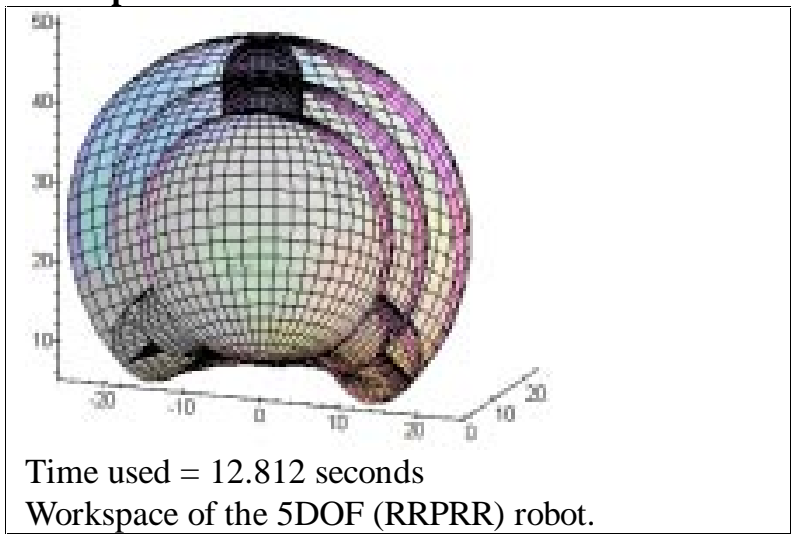

\section{CONCLUSIONS}

The algorithm and experimental computer code presented in this paper are a culmination of efforts to study formulations for analyzing and visualizing multivariate solids. It was shown that the method encompasses geometric entities defined in both parametric and implicit forms. It was also shown that the rigorous mathematical formulation using manifold stratification is capable of addressing multivariate solid representations. Unilateral constraints imposed on the solid were also taken into consideration. Exact representation of the solid is achieved and it was shown that the methodology is suitable for computer implementation. While the computer code developed using Mathematica ${ }^{\circledR}$ is not optimized for commercial use, it is an illustration of the potential of this method for efficient implementation. The ultimate goal is to implement the formulation as an integral part of commercial $\mathrm{CAD}$ systems or as a stand-alone module. Moreover, to address the visualization of the multivariate freeform solid.

Computational time measured for various examples using the Mathematica ${ }^{\circledR}$ experimental code provides an indication of the efficiency of the code, in view of implementing such algorithms in a more appropriate computer language or hardware.

Results are shown that demonstrate the robustness of the method in visualizing the multivariate solid. The principal contribution of this method is characterized by its ability to obtain an exact representation for visualizatin purposes, and in its ability to obtain closed-form equations of its boundary.

\section{REFERENCES}

Abdel-Malek, K. and Othman, S., 1999 (in press) "Multiple Sweeping using the Denavit-Hartenberg Representation Method," Computer-Aided Design, Vol. 31, pp. 567-583.

Abdel-Malek, K. and Yeh, H.J., 1997a "Geometric Representation of the Swept Volume Using Jacobian Rank-Deficiency Conditions," Computer Aided Design, Vol. 29, No. 6, pp. 457-468.

Abdel-Malek, K. and Yeh, H.J., 1997b "Analytical Boundary of the Workspace for General Three Degree-of-Freedom
Mechanisms," International Journal of Robotics Research. Vol. 16, No. 2, pp. 198-213.

Abdel-Malek, K., Seaman, W., and Yeh, H.J., 1999a (Submitted) "NC Verification of up to 5 Axis Machining Processes Using Manifold Stratification" ASME Journal of Manufacturing Science and Engineering.

Abdel-Malek, K., Yang, J., and Blackmore, D 1999c (submitted) "On Swept Volume Formulations: Implicit Surfaces," Computer Aided Design

Abdel-Malek, K., Yeh, H.J., and Khairallah, N., 1999b "Workspace, Void, and Volume Determination of the General 5DOF Manipulator, Mechanics of Structures and Machines, Vol. 27, No. 1, 91-117.

Abdel-malek, K., Yeh, H.J., and Othman, S., 1998, " Swept Volumes: Void and Boundary Identification," Computer Aided Design, Vol. 30, No. 13, pp. 1009-1018

Abdel-Malek, K., Yeh, H.J., and Rouwady, R., 1997a "A Unified Formulation for Swept Volume and Robot Workspace Analysis," Proceedings of the ASME Design Automation Conference, Sacramento, CA, Sep. pp14-17.

Abrams, S and Allen, P K, 1995, "Swept Volumes and their use in viewpoint computation in robot work-cells" Proceedings of the IEEE International Symposium on Assembly and Task Planning (1995) pp188-193.

Ahn, J.W., Kim, M.S., and Lim, S.B., 1993, Approximate General Sweep Boundary of a 2D Curved Object, Comput. Visi, Graps, Img Prcs, Vol. 55(2), pp. 98-128.

Blackmore, D and Leu, M C, 1992, 'Analysis of Swept Volume via Lie Groups and Differential Equations' Int. Journal of Robotics Research Vol. 11(6) (1992) pp516-537.

Blackmore, D, Jiang, H, and Leu, M C Modeling of Swept Solids Using Sweep Differential Techniques' Proc. 4th IFIP WG5.2 Workshop Geometric Modeling in ComputerAided Design, Rensselaerville, NU (1992).

Blackmore, D, Leu, M C, and Frank, S 'Analysis and Modeling of Deformed Swept Volumes' Computer Aided Design Vol. 25(4) (1994), pp. 315-326.

Blackmore, D, Leu, MC, Wang, LP, Jiang, H, 1997, "Swept Volumes: a retrospective and prospective view," Neural parallel and Scientific Computations, Vol. 5, pp81-102.

Blackmore, D.; Leu, M.C.; Wang, L.P., 1997, "Sweep-envelope differential equation algorithm and its application to NC machining verification", Comp Aided Des 29(9), 629-637.

Boussac, S and Crosnier, A, 1996, "Swept volumes generated from deformable objects application to NC verification", Proc 13th IEEE Int Conf on Rob and Aut. Part 2 (of 4) Apr 22-28, v 2, Minneapolis, MN, pp. 1813-1818

Bronsvoort, W., Van Nieuwenhuizen, P., and Post, F., 1989, Display of Profiled Sweep Objects. The Visual Computer, 1989, Vol. 5(3), pp. 147-157.

Coquillart, S., 1987, A Control Point Based Sweeping Technique. IEEE Computer Graphics and Applications, 1987, Vol. 7(11), pp. 36-44. 
Ganter, M A, Storti, D W, and Ensz, M T, 1993, "On Algebraic Methods for Implicit Swept Solids with Finite Extent" ASME DE Vol. 65(2) pp. 389-396.

Guillemin, V. and Pollack, A., 1974, Differential Topology, Prentice-Hall.

Hu, Z J and Ling, Z K, 1994, "Generating Swept Volumes with Instantaneous Screw Axes" Proceedings of the 1994 ASME Design Technical Conference, Part 1, Minneapolis, MN (1994), DE Vol 70(1) pp.7-14.

$\mathrm{Hu}, \mathrm{Z} \mathrm{J}$, and Ling, Z K, 1996, Swept volumes generated by the natural quadric surfaces. Computers and Graphics, Vol. 20, No. 2, pp. 263-274.

Ilies, H.T. and Shapiro, V., 1999, "The Dual of Sweep", Computer Aided Design Vol. 31, pp.185-201.

Jerard, R B and Drysdale, R L, 1988, "Geometric Simulation of Numerical Control Machinery" ASME Computers Engineer Vol 2 pp129-136.

Joy, KI and Duchaineau, MA 1999 "Boundary Determination for Trivariate Solids", Proceedings of the 1999 Pacific Graphics Confernece, Seoul, Korea, October 5-7, 1999.

Juttler, B and Wagner, M.G., 1996, "Computer aided design with spatial rational B-spline motions," Journal of Mechanical Design, 118, pp.193-201.

Juttler, B and Wagner, M.G., 1999, "Rational motion-based surface generation," Compt Aided Design, 31, 203-213.

Kieffer, J and Litvin, F L, 1991, "Swept Volume Determination and Interference Detection for Moving 3-D Solids" ASME, Journal of Mechanical Design Vol 113 (1990) pp456-463.

Klok, F, 1986, "Two Moving Coordinate Frames for Sweeping Along a 3D Trajectory" Computer-Aided Geometric Design, Vol. 3, pp. 217-229

Leu, M C, Park, S H, and Wang, K K, 1986, "Geometric Representation of Translational Swept Volumes and Its Applications" ASME Journal of Engineering for Industry, Vol 108 (1986) pp. 113-119.

Liang, X; Xiao, T; Han, X; Ruan, JX, 1997, Simulation Software GNCV of NC Verification, Author Affiliation: ICIPS Proceedings of the 1997 IEEE International Conference on Intelligent Processing Systems, Part 2 Oct 28-31, v 2, Beijing, China pp. 1852-1856.

Liu, Ch.; Esterling, D.M.; Fontdecaba, J.; Mosel, E, 1996, "Dimensional verification of NC machining profiles using extended quadtrees", Comp Aided Des 28(11), 845-852

Lu, Y.C., 1976, Singularity Theory and an Introduction to Catastrophe Theory, Springer-Verlag.

Madrigal, C and Joy, KI 1999 "Generating the Envelope of a Swept Trivariate Solid," Proceedings of the IASTED International Conference on Computer Graphics and Imaging, Palm Springs, California, October 25-27, 1999.

Martin, R R and Stephenson, P C, 1990, "Sweeping of threedimensional Objects" Comp Aided Des 22(4), 223-234.

Menon, J.P.; Voelcker, H.B. 1992, "Toward a comprehensive formulation of $\mathrm{NC}$ verification as a mathematical and computational problem", Proc 1992 Winter Annual Meeting of ASME, 59, Anaheim, CA, pp. 147-164

Parida, L and Mudur, S P, 1994, "Computational Methods for Evaluating Swept Object Boundaries" Visual Computer Vol 10(5) (1994) pp. 266-276.

Sambandan, K and Wang, K K, 1989, "Five-axis Swept Volumes for Graphic NC Simulation and Verification" ASME DE 19(1) pp. 143-150.

Schroeder, W J, Lorensen, W E, and Linthicum, S, 1994, "Implicit Modeling of Swept Surfaces and Volumes" Proc. IEEE Visualiz Conf, Los Alamitos, CA, pp. 40-45.

Spivak, M. 1968, Calculus on Manifolds, Benjamin/Cummeings.

Voelker, H B and Hunt, W A, 1985, "The Role of Solid Modeling in Machining Process Modeling and NC Verification" SAE Tech. Paper \#810195.

Wang, W P and Wang, K K, 1986, "Geometric Modeling for Swept Volume of Moving Solids" IEEE Computer Graphics and Applications, Vol. 6(12), pp. 8-17.

Weld, J D and Leu, M C, 1991, "Geometric Representation of Swept Volumes With Application to Polyhedral Objects" Int Journal of Robotics Research Vol9(5) pp. 105-117.

Whitney, H., 1965, "Tangents to an Analytic Variety," Ann. of Math., Vol. 81, pp. 496-540. 